\title{
A Summary of Replacement Policies with Number of Failures
}

\author{
Xufeng Zhao \\ College of Economics and Management \\ Nanjing University of Aeronautics and Astronautics, Nanjing 211106, China \\ Corresponding author: xz.cem@nuaa.edu.cn \\ Cunhua Qian \\ School of Economics and Management \\ Nanjing Tech University, Nanjing 211800, China \\ Syouji Nakamura \\ Department of Life Management \\ Kinjo Gakuin University, Nagoya 463-8521, Japan \\ Toshio Nakagawa \\ Department of Business Administration \\ Aichi Institute of Technology, Toyota 470-0392, Japan
}

(Received March 22, 2017; Accepted September 27, 2017)

\begin{abstract}
Periodic replacement models with minimal repairs have been studied extensively. In order to prevent high repair costs after failures, this paper considers the number of failures as another decision variable for replacement policies. We begin with the standard periodic replacement model, and then summarize several extended replacement models with number of failures, using the respective assumptions of whichever occurs first and last, and replacing over planned measure, respectively. Optimum policies of replacement times $T$, number of periodic times $N$, and number of failures $K$ are discussed analytically. From the optimization of the integrated models of $T, N$ and $K$, comparisons among these policies are made in analytical ways to determine which policy should be more economical.
\end{abstract}

Keywords- Minimal repair, Periodic replacement, Replacement last, Random failure, Cumulative hazard rate.

\section{Introduction}

Maintenance policies are commonly performed at periodic times in applications. For example, maintenance in total productive maintenance (TPM) consists of periodical inspection, cleaning and servicing equipment and replacing deteriorated units to prevent serious breakdown problems (Venkatesh, 2007). The original model of periodic replacement was formulated by using the cumulative hazard rate, in which minimal repairs can fix failures without disturbing the failure rate (Barlow and Proschan, 1996; Nakagawa, 2005). Other theoretical research works on periodic patterns in maintenance plans have been studied extensively (Taghipour et al., 2010; Huynh et al., 2011; Taghipour and Banjevic, 2012; Golmakani and Moakedi, 2012; Wang and Banjevic, 2012; Taghipour and Kassaei, 2015; Kim et al., 2015).

Minimal repairs that cost less are always considered at minor failures for the large and complex systems with many kinds of units (Barlow and Proschan, 1996). Models for repairable system subjected to minimal repair (Pulcini, 2003), imperfect repair considering time-dependent repair effectiveness (Fuqing and Kumar, 2012), age-based replacement with repair for shocks and 
International Journal of Mathematical, Engineering and Management Sciences

Vol. 3, No. 2, 136-150, 2018

https://dx.doi.org/10.33889/IJMEMS.2018.3.2-011

degradation (Huynh et al., 2012), post-warranty maintenance with repair time threshold (Park et al., 2013), etc., have been studied extensively. Most recently, random working models with replacement and minimal repair (Zhao et al., 2016), and a case study of periodic maintenance policies for off-road engines has been surveyed (Toledo et al., 2016).

In order to minimize the repair and replacement cost for a long run and maximize the utilization of the operating unit, this paper begins with the standard periodic replacement model with minimal repairs (Nakagawa, 2005) and then summarizes several extended replacement policies with failures, using the assumptions of replacement first (Nakagawa, 2005), replacement last (Zhao and Nakagawa, 2012), and replacement overtime (Nakagawa and Zhao, 2015; Zhao et al., 2015). That is, assumptions of whichever occurs first and last, and replacing over planned measure will be taken into considerations for replacement policies, respectively. It will show that the extended models include the standard periodic replacement with minimal repairs. It has been indicated in (Zhao and Nakagawa, 2012; Nakagawa and Zhao, 2015; Zhao et al., 2015) that the last and overtime modes in replacement policies could let the unit operate for longer time with minimum replacement cost rates, which will also be discussed and compared with replacement first in this paper.

Throughout the paper, we assume that failures occur randomly at a non-homogeneous Poisson process with a hazard rate $H(t)$, then the probability that a number $j$ of failures occur during time interval $[0, t]$ is

$$
p_{j}(t) \equiv \frac{H(t)^{j}}{j !} e^{-H(t)} \quad(j=0,1,2, \ldots)
$$

Denote that $P_{j}(t) \equiv \sum_{i=j}^{\infty} p_{i}(t), \bar{P}_{j}(t) \equiv 1-P_{j}(t)=\sum_{i=0}^{j-1} p_{i}(t)$, then $P_{0}(t) \equiv 1$ and $\bar{P}_{0}(t)=$ 0 .

In addition, the failure distribution is obtained as $F(t) \equiv \sum_{j=1}^{\infty} p_{j}(t)=1-p_{0}(t)=1-e^{-H(t)}$ with mean time $\mu \equiv \int_{0}^{\infty} \bar{F}(t) d t$, and the failure rate is denoted as $h(t) \equiv d H(t) / d t$, where $h(t)$ increases with $t$ from 0 to $h(\infty) \equiv \lim _{t \rightarrow \infty} h(t)$. We suppose the failures are fixed by minimal repairs without disturbing the failure rate. In order to prevent high repair costs after failures, we begin with the standard periodic model with minimal repairs in Section 2, and then summarize several extended replacement models with number of failures from Section 3.

\section{Standard Periodic Model}

Suppose the unit is replaced at periodic times $n T(n=1,2, \ldots ; 0<T<\infty)$. Then (Nakagawa, 2005)

$$
C_{T}(T)=\frac{c_{T}+c_{M} H(T)}{T},
$$

where $c_{T}=$ replacement cost at each periodic time and $c_{M}=$ cost of minimal repair at each failure. Clearly,

$$
C_{T}(0) \equiv \lim _{T \rightarrow 0} C_{T}(T)=\infty, C_{T}(\infty) \equiv \lim _{T \rightarrow \infty} C_{T}(T)=c_{M} h(\infty)
$$


International Journal of Mathematical, Engineering and Management Sciences

Vol. 3, No. 2, 136-150, 2018

https://dx.doi.org/10.33889/IJMEMS.2018.3.2-011

Optimum $T^{*}$ to minimize $C_{T}(T)$ satisfies

$T h(T)-H(T)=\frac{c_{T}}{c_{M}}$, i.e., $\int_{0}^{T} t d h(t)=\frac{c_{T}}{c_{M}}$.

The resulting cost rate is

$C_{T}\left(T^{*}\right)=c_{M} h\left(T^{*}\right)$

This optimum policy indicates that minimal repairs are made if some failures occur during $\left(0, T^{*}\right)$, and the unit is replaced at time $T^{*}$.

When $H(t)=t^{m} \quad(m>1)$, optimum $T^{*}$ is given by a solution of $(m-1) T^{m}=c_{T} / c_{M}$. Table 1 presents optimum $T^{*}$ and its cost rate for $m$ and $c_{T} / c_{M}$. This shows that $T^{*}$ increases with $c_{T} / c_{M}$ and decreases with $m$, and its cost rate $C_{T}\left(T^{*}\right)$ increases with replacement $\operatorname{cost} c_{T}$ and the failure numbers for repairs.

Table 1. Optimum $T^{*}$ and its cost rate when $H(t)=t^{m}$

\begin{tabular}{|c|c|c|c|c|}
\hline \multirow{2}{*}{$c_{T} / c_{M}$} & \multicolumn{3}{|c|}{$m=2.0$} & \multicolumn{2}{c|}{$m=3.0$} \\
\cline { 2 - 5 } & $T^{*}$ & $C_{T}\left(T^{*}\right) / c_{M}$ & $T^{*}$ & $C_{T}\left(T^{*}\right) / c_{M}$ \\
\hline 2.0 & 1.41 & 2.83 & 1.00 & 3.00 \\
\hline 3.0 & 1.73 & 3.46 & 1.14 & 3.93 \\
\hline 4.0 & 2.00 & 4.00 & 1.26 & 4.76 \\
\hline 5.0 & 2.24 & 4.47 & 1.36 & 5.53 \\
\hline 6.0 & 2.45 & 4.90 & 1.44 & 6.24 \\
\hline 7.0 & 2.65 & 5.29 & 1.52 & 6.91 \\
\hline 8.0 & 2.83 & 5.66 & 1.59 & 7.56 \\
\hline 9.0 & 3.00 & 6.00 & 1.65 & 8.18 \\
\hline 10.0 & 3.16 & 6.32 & 1.71 & 8.77 \\
\hline
\end{tabular}

\section{Failure Over Periodic Time}

As the first extension of the standard periodic replacement model in Section 2, we suppose the unit cannot be replaced strictly at periodic times, but is replaced at the first failure over the above each periodic time $n T \quad(n=1,2, \ldots ; 0<T<\infty)$. Now the problem become to reconsider the periodic interval $T$ with over time mode. Then (Nakagawa and Zhao, 2015)

$C_{O}(T)=\frac{c_{O}+c_{M}[H(T)+1]}{T+1 / Q(T)}$

where $c_{O}=$ replacement cost over each time $T$, and

$$
Q(T) \equiv \frac{1}{\int_{T}^{\infty} e^{-[H(t)-H(T)]} d t}=\frac{\bar{F}(T)}{\int_{T}^{\infty} \bar{F}(t) d t} \geq h(T)
$$


International Journal of Mathematical, Engineering and Management Sciences

Vol. 3, No. 2, 136-150, 2018

https://dx.doi.org/10.33889/IJMEMS.2018.3.2-011

Clearly, (5) and (1) have similar forms.

When $T \rightarrow \infty$,

and when $T \rightarrow 0$,

$$
C_{O}(\infty) \equiv \lim _{T \rightarrow \infty} C_{O}(T)=c_{M} h(\infty)
$$

$C_{O}(0) \equiv \lim _{T \rightarrow 0} C_{O}(T)=\frac{c_{O}+c_{M}}{\mu}$

Differentiating $C_{O}(T)$ with respect to $T$ and setting it equal to zero,

$T Q(T)-H(T)=\frac{c_{o}}{c_{M}}$, i.e., $\int_{0}^{T}[Q(T)-h(t)] d t=\frac{c_{o}}{c_{M}}$.

Therefore, the following optimum policies are given:

a) If $\int_{0}^{\infty} t d h(t)>c_{O} / c_{M}$, then there exists a unique $T_{O}^{*}\left(0<T_{O}^{*}<\infty\right)$ in (7) to minimize $C_{O}(T)$, and

$C_{O}\left(T_{O}^{*}\right)=c_{M} Q\left(T_{o}^{*}\right)=\frac{c_{O}+c_{M} H\left(T_{O}^{*}\right.}{T_{O}^{*}}$.

b) If $\int_{0}^{\infty} t d h(t) \leq c_{O} / c_{M}, T_{O}^{*}=\infty$, and the cost rate has been given in (2).

Further, it can be found that $T_{O}^{*} \leq T$ as $Q(T) \geq h(T)$ in (3) and (7). Thus, comparing of (1) and (8), when $c_{O}=c_{T}$, the standard periodic replacement is more economical.

When $H(t)=t^{m}$, Table 2 presents optimum optimum $T_{O}^{*}$ and its cost rate for $m$ and $c_{O} / c_{M}$. This indicates that $T_{O}^{*}<T$ and $C_{O}\left(T_{O}^{*}\right)>C_{T}\left(T^{*}\right)$ when $c_{O}=c_{T}$. However, the differences between two expected cost rates become smaller as $c_{O} / c_{M}$ are larger.

Table 2. Optimum $T_{O}^{*}$ and its cost rate when $H(t)=t^{m}$

\begin{tabular}{|c|c|c|c|c|}
\hline \multirow{2}{*}{$c_{O} / c_{M}$} & \multicolumn{2}{|c|}{$m=2.0$} & \multicolumn{2}{c|}{$m=3.0$} \\
\cline { 2 - 5 } & $T_{O}^{*}$ & $C_{O}\left(T_{O}^{*}\right) / c_{M}$ & $T_{O}^{*}$ & $C_{O}\left(T_{O}^{*}\right) / c_{M}$ \\
\hline 2.0 & 1.15 & 2.89 & 0.78 & 3.18 \\
\hline 3.0 & 1.51 & 3.50 & 0.96 & 4.05 \\
\hline 4.0 & 1.81 & 4.02 & 1.10 & 4.84 \\
\hline 5.0 & 2.07 & 4.49 & 1.22 & 5.58 \\
\hline 6.0 & 2.31 & 4.91 & 1.33 & 6.28 \\
\hline 7.0 & 2.53 & 5.30 & 1.42 & 6.94 \\
\hline 8.0 & 2.74 & 5.66 & 1.51 & 7.58 \\
\hline 9.0 & 2.93 & 6.00 & 1.59 & 8.19 \\
\hline 10.0 & 3.12 & 6.33 & 1.66 & 8.78 \\
\hline
\end{tabular}


International Journal of Mathematical, Engineering and Management Sciences

Vol. 3, No. 2, 136-150, 2018

https://dx.doi.org/10.33889/IJMEMS.2018.3.2-011

\section{Failure Number}

In order to extend the following replacement policies with failures, we firstly in this section consider the case when only failure number is used for replacement decision. That is, the unit is replaced at a number $K(K=1,2, \ldots)$ of failures. Then (Nakagawa, 2005; Nakagawa and Zhao, 2015)

$$
C_{N}(K)=\frac{c_{K}+c_{M} K}{\int_{0}^{\infty} \overline{P_{K}}(t) d t}(K=1,2, \ldots),
$$

where $c_{K}=$ replacement cost at failure $K$. Clearly,

$$
C_{N}(\infty) \equiv \lim _{K \rightarrow \infty} C_{N}(K)=c_{M} h(\infty)
$$

in (2), and

$$
C_{N}(1)=C_{O}(0)=\frac{c_{K}+c_{M}}{\mu}
$$

in (6) when $c_{O}=c_{K}$. Forming the inequality $C_{N}(K+1)-C_{N}(K) \geq 0$,

$$
\frac{\int_{0}^{\infty} P_{K}(t) d t}{\int_{0}^{\infty} p_{K}(t) d t}-K \geq \frac{c_{K}}{c_{M}} \text {. }
$$

The left-hand side of (10) increases with $K$ (Nakagawa, 2005; Nakagawa, and Zhao, 2015). Letting $L_{1}(K)$ be the left-hand side of $(10)$, if $L_{1}(\infty)>c_{K} / c_{M}$, then there exists a unique $K^{*}\left(1 \leq K^{*}<\infty\right)$ in $(10)$ to minimize $C_{N}(K)$, and the resulting cost rate is

$$
\frac{c_{M}}{\int_{0}^{\infty} p_{K^{*}-1}(t) d t}<C_{N}\left(K^{*}\right) \leq \frac{c_{M}}{\int_{0}^{\infty} p_{K^{*}}(t) d t} \text {. }
$$

When $H(t)=t^{m}$, Table 3 presents optimum optimum $K^{*}$ and its cost rate for $m$ and $c_{K} / c_{M}$. In this case, optimum $K^{*}$ is given by $K^{*}=\left[\left(c_{K}-c_{M}\right) /(m-1) c_{M}\right]+1$, where $[x]$ means the greatest integer contained in $x$. It is nature that $K^{*}$ can be increasing when the repair cost $c_{M}$ decrease and the cost rate $C_{N}\left(K^{*}\right)$ increases with the number of failures.

Table 3. Optimum $K^{*}$ and its cost rate when $H(t)=t^{m}$

\begin{tabular}{|c|c|c|c|c|}
\hline \multirow{2}{*}{$c_{K} / c_{M}$} & \multicolumn{2}{|c|}{$m=2.0$} & \multicolumn{2}{c|}{$m=3.0$} \\
\cline { 2 - 5 } & $K^{*}$ & $C_{N}\left(K^{*}\right) / c_{M}$ & $K^{*}$ & $C_{N}\left(K^{*}\right) / c_{M}$ \\
\hline 2.0 & 2 & 2.89 & 2 & 3.36 \\
\hline 3.0 & 3 & 3.50 & 2 & 4.20 \\
\hline 4.0 & 5 & 4.02 & 3 & 5.04 \\
\hline 5.0 & 5 & 4.49 & 3 & 5.76 \\
\hline 6.0 & 6 & 4.91 & 3 & 6.48 \\
\hline 7.0 & 7 & 5.30 & 4 & 7.13 \\
\hline 8.0 & 8 & 5.66 & 4 & 7.77 \\
\hline 9.0 & 10 & 6.00 & 5 & 8.37 \\
\hline 10.0 & 10 & 6.33 & 6 & 8.97 \\
\hline
\end{tabular}


International Journal of Mathematical, Engineering and Management Sciences

Vol. 3, No. 2, 136-150, 2018

https://dx.doi.org/10.33889/IJMEMS.2018.3.2-011

\section{Next Periodic Time}

Suppose the unit is replaced at the next periodic time $(n+1) T$ over periodic time $n T$ ( $n=$ $0,1,2, \ldots ; 0<T<\infty)$ when more than $K(K=1,2, \ldots)$ failures have arrived, and we obtain an optimum $K$ for given. Then (Nakagawa, 2005)

$C_{P}(K, T)=\frac{c_{T}+c_{M} \sum_{n=0}^{\infty}[H((n+1) T)-H(n T)] \bar{P}_{K}(n T)}{T \sum_{n=0}^{\infty} \bar{P}_{K}(n T)} \quad(K=1,2, \ldots)$.

Forming the inequality $C_{P}(K+1, T)-C_{P}(K, T) \geq 0$,

$Q_{1}(K, T) \sum_{n=0}^{\infty} \bar{P}_{K}(n T)-\sum_{n=0}^{\infty}[H((n+1) T)-H(n T)] \bar{P}_{K}(n T) \geq \frac{c_{T}}{c_{M}}$,

where

$Q_{1}(K, T) \equiv \frac{\sum_{n=0}^{\infty}[H((n+1) T)-H(n T)] p_{K}(n T)}{\sum_{n=0}^{\infty} \bar{P}_{K}(n T)}$.

Thus, the left-hand side $L_{2}(K, T)$ of (13) increases strictly with $K$. If $L_{2}(K, T)>c_{T} / c_{M}$, then there exists a unique and minimum $K_{P}^{*}\left(1 \leq K_{P}^{*}<\infty\right)$ in $(13)$ to minimize $C_{P}(K, T)$, and

$c_{M} Q_{1}\left(K_{P}^{*}-1, T\right)<C_{P}\left(K_{P}^{*}, T\right) \leq c_{M} Q_{1}\left(K_{P}^{*}, T\right)$.

When $H(t)=t^{m}$, Table 4 presents optimum $K_{P}^{*}$ and its cost rate for $m$ and $c_{T} / c_{M}$, and it shows the same tendencies with Table 4.

Table 4. Optimum $K_{P}^{*}$ and its cost rate when $H(t)=t^{m}$ and $T=0.1$

\begin{tabular}{|c|c|c|c|c|}
\hline \multirow{2}{*}{$c_{T} / c_{M}$} & \multicolumn{2}{|c|}{$m=2.0$} & \multicolumn{2}{c|}{$m=3.0$} \\
\cline { 2 - 5 } & $K_{P}^{*}$ & $C_{P}\left(K_{P}^{*}, T\right) / c_{M}$ & $K_{P}^{*}$ & $C_{P}\left(K_{P}^{*}, T\right) / c_{M}$ \\
\hline 2.0 & 2 & 3.00 & 1 & 3.42 \\
\hline 3.0 & 3 & 3.60 & 1 & 4.40 \\
\hline 4.0 & 4 & 4.12 & 2 & 5.03 \\
\hline 5.0 & 5 & 4.58 & 3 & 5.78 \\
\hline 6.0 & 6 & 5.00 & 3 & 6.47 \\
\hline 7.0 & 7 & 5.38 & 4 & 7.14 \\
\hline 8.0 & 8 & 5.74 & 4 & 7.77 \\
\hline 9.0 & 19 & 6.08 & 5 & 8.39 \\
\hline 10.0 & 10 & 6.40 & 6 & 9.00 \\
\hline
\end{tabular}

\section{Time and Failure Number}

\subsection{Replacement First}

Suppose the unit is replaced at planned time $T(0<T \leq \infty)$ or at failure number $K(1 \leq$ $K \leq \infty)$, whichever occurs first. Then

$C_{F}(T, K)=\frac{c_{T} \bar{P}_{K}(T)+c_{K} P_{K}(T)+c_{M} \int_{0}^{T} \bar{P}_{K}(t) h(t) d t}{\int_{0}^{T} \bar{P}_{K}(t) d t}$,

clearly, $C_{F}(T, \infty)=C_{T}(T)$ in $(1)$ and $C_{F}(\infty, K)=C_{N}(K)$ in (9). 
International Journal of Mathematical, Engineering and Management Sciences

Vol. 3, No. 2, 136-150, 2018

https://dx.doi.org/10.33889/IJMEMS.2018.3.2-011

When $c_{T}=c_{K}$, differentiating $C_{F}(T, K)$ with respect to $T$ and setting it equal to zero,

$\int_{0}^{T} \bar{P}_{K}(t)[h(T)-h(t)] d t=\frac{c_{T}}{c_{M}}$.

Letting $L_{3}(T, K)$ be the left-hand side of $(16), L_{3}(T, K)$ increases strictly with $T$ from 0 . Thus, if $L_{3}(\infty, K)>c_{T} / c_{M}$, then there exists a unique $T_{F}^{*}\left(0<T_{F}^{*}<\infty\right)$ which satisfies (16), and

$C_{F}\left(T_{F}^{*}, K\right)=c_{M} h\left(T_{F}^{*}\right)$

The optimum policy to minimize $C_{F}(T, K)$ is $\left(T_{F}^{*}=T^{*}, K_{F}^{*}=\infty\right)$. The optimum policy indicates that replacement done at time $T$ is more economical than that at failure $K$.

Next, we find optimum $K_{F}^{*}$ to minimize $C_{F}(T, K)$ for given $T$. Forming the inequality $C_{F}(T, K+$ 1) $-C_{F}(T, K) \geq 0$,

$H_{1}(T, K) \int_{0}^{T} \bar{P}_{K}(t) d t-\int_{0}^{T} \bar{P}_{K}(t) h(t) d t \geq \frac{c_{T}}{c_{M}}$,

where

$H_{1}(T, K)=\frac{\int_{0}^{T} H(t)^{K} d F(t)}{\int_{0}^{T} H(t)^{K} \bar{F}(t) d t}$

Therefore, if $T \leq T^{*}$, then $K_{F}^{*}=\infty$, and conversely, if $T>T^{*}$, then optimum $K_{F}^{*}\left(1 \leq K_{F}^{*}<\right.$ $\infty)$ which satisfies (18) exists.

\subsection{Replacement Last}

Suppose the unit is replaced at planned time $T(0 \leq T \leq \infty)$ or at failure number $K(0 \leq K \leq$ $\infty)$, whichever occurs last. The mean time to replacement is

$T P_{K}(T)+\int_{T}^{\infty} t d P_{K}(t)=T+\int_{T}^{\infty} \bar{P}_{K}(t) d t$

The expected number of failures until replacement is

$H(T)+\int_{T}^{\infty} \bar{P}_{K}(t) h(t) d t$

which agrees with (19) when $h(t)=1$. Thus

$C_{L}(T, K)=\frac{c_{T} P_{K}(T)+c_{K} \bar{P}_{K}(T)+c_{M}\left[H(T)+\int_{T}^{\infty} \bar{P}_{K}(t) h(t) d t\right]}{T+\int_{T}^{\infty} \bar{P}_{K}(t) d t}$.

Clearly, $C_{L}(0, K)=C_{F}(\infty, K)=C_{N}(K)$ and $C_{L}(T, 0)=C_{F}(T, \infty)=C_{T}(T)$.

When $c_{T}=c_{K}$, differentiating $C_{L}(T, K)$ with respect to $T$ and setting it equal to zero,

$\int_{0}^{T}[h(T)-h(t)] d t-\int_{T}^{\infty} \bar{P}_{K}(t)[h(t)-h(T)] d t=\frac{c_{T}}{c_{M}}$. 
International Journal of Mathematical, Engineering and Management Sciences

Vol. 3, No. 2, 136-150, 2018

https://dx.doi.org/10.33889/IJMEMS.2018.3.2-011

There exists a unique $T_{L}^{*}\left(0<T_{L}^{*}<\infty\right)$ which satisfies (22), and the resulting cost rate is

$C_{L}\left(T_{L}^{*}, K\right)=c_{M} h\left(T_{L}^{*}\right)$

The optimum policy to minimize $C_{L}(T, K)$ is $\left(T_{L}^{*}=T^{*}, K_{L}^{*}=\infty\right)$. The optimum policy indicates that replacement done at time $T$ is more economical than that at failure $K$.

Next, we find optimum $K_{L}^{*}$ to minimize $C_{L}(T, K)$ for given $T$. Forming the inequality $C_{L}(T, K+1)-C_{L}(T, K) \geq 0$,

$\int_{0}^{T}\left[\widetilde{H}_{1}(T, K)-h(t)\right] d t-\int_{T}^{\infty} \bar{P}_{K}(t)[h(t)-h(T)] d t \geq \frac{c_{T}}{c_{M}}$,

where

$\widetilde{H}_{1}(T, K) \equiv \frac{\int_{T}^{\infty} H(t)^{K} d F(t)}{\int_{T}^{\infty} H(t)^{K} \bar{F}(t) d t}$.

Thus, the left-hand side of (24) increases strictly from that of (7). Therefore, if $T \geq T_{O}^{*}$, then $K_{L}^{*}=0$, and conversely, if $T<T_{O}^{*}$, then optimum $K_{L}^{*}\left(1 \leq K_{L}^{*}<\infty\right)$ exists.

The following results are obtained by comparing the policies of periodic replacement, replacement first and last in Sections 2 and 6:

a) If $T<T_{O}^{*}$, then replacement last should be adopted.

b) If $T_{O}^{*} \leq T \leq T^{*}$, then standard periodic replacement should be adopted.

c) If $T>T^{*}$, then replacement first should be adopted.

\subsection{Numerical Examples}

Table 5 presents optimum $T_{F}^{*}$ and $T_{L}^{*}$ and their cost rates for given $K$ when $H(t)=t^{2.0}$. Table 6 presents optimum $K_{F}^{*}$ and $K_{L}^{*}$ and their cost rates for given $T$ when $H(t)=t^{2.0}$. In Table 5, when $K=5$, it shows that $T_{F}^{*}<T_{L}^{*}$ and $C_{F}\left(T_{F}^{*}, K\right)<C_{L}\left(T_{L}^{*}, K\right)$ for $c_{T} / c_{M} \leq 4.0$. In Table 6, it shows that $C_{F}\left(T, K_{F}^{*}\right)>C_{L}\left(T, K_{L}^{*}\right)$ for $K_{F}^{*}=\infty$ and $C_{F}\left(T, K_{F}^{*}\right)<C_{L}\left(T, K_{L}^{*}\right)$ for 0 .

Table 5. Optimum $T_{F}^{*}$ and $T_{L}^{*}$ and their cost rates for given $K$ when $H(t)=t^{2.0}$

\begin{tabular}{|c|c|c|c|c|c|c|c|c|}
\hline \multirow{2}{*}{$c_{T} / c_{M}$} & \multicolumn{9}{|c|}{$K=1$} & \multicolumn{4}{c|}{$K=5$} \\
\cline { 2 - 9 } & $T_{F}^{*}$ & $C_{F}\left(T_{F}^{*}, K\right) / c_{M}$ & $T_{L}^{*}$ & $C_{L}\left(T_{L}^{*}, K\right) / c_{M}$ & $T_{F}^{*}$ & $C_{F}\left(T_{F}^{*}, K\right) / c_{M}$ & $T_{L}^{*}$ & $C_{L}\left(T_{L}^{*}, K\right) / c_{M}$ \\
\hline 2.0 & 1.69 & 3.38 & 1.42 & 2.84 & 1.42 & 2.83 & 1.60 & 3.20 \\
\hline 3.0 & 2.26 & 4.51 & 1.73 & 3.47 & 1.74 & 3.47 & 1.83 & 3.65 \\
\hline 4.0 & 2.82 & 5.64 & 2.00 & 4.00 & 2.02 & 4.03 & 2.05 & 4.09 \\
\hline 5.0 & 3.39 & 6.77 & 2.24 & 4.47 & 2.27 & 4.54 & 2.26 & 4.52 \\
\hline 6.0 & 3.95 & 7.90 & 2.45 & 4.90 & 2.51 & 5.02 & 2.46 & 4.92 \\
\hline 7.0 & 4.51 & 9.03 & 2.65 & 5.29 & 2.75 & 5.49 & 2.65 & 5.30 \\
\hline 8.0 & 5.08 & 10.16 & 2.83 & 5.66 & 2.98 & 5.96 & 2.83 & 5.66 \\
\hline 9.0 & 5.64 & 11.28 & 3.00 & 6.00 & 3.21 & 6.42 & 3.00 & 6.00 \\
\hline 10.0 & 6.21 & 12.41 & 3.16 & 6.32 & 3.44 & 6.88 & 3.16 & 6.33 \\
\hline
\end{tabular}


International Journal of Mathematical, Engineering and Management Sciences

Vol. 3, No. 2, 136-150, 2018

https://dx.doi.org/10.33889/IJMEMS.2018.3.2-011

Table 6. Optimum $K_{F}^{*}$ and $K_{L}^{*}$ and their cost rates for given $T$ when $H(t)=t^{2.0}$

\begin{tabular}{|c|c|c|c|c|c|c|c|c|}
\hline \multirow{2}{*}{$c_{T} / c_{M}$} & \multicolumn{9}{|c|}{$T=1.0$} & \multicolumn{4}{c|}{$T=5.0$} \\
\cline { 2 - 9 } & $K_{F}^{*}$ & $C_{F}\left(T, K_{F}^{*}\right) / c_{M}$ & $K_{L}^{*}$ & $C_{L}\left(T, K_{L}^{*}\right) / c_{M}$ & $K_{F}^{*}$ & $C_{F}\left(T, K_{F}^{*}\right) / c_{M}$ & $K_{L}^{*}$ & $C_{L}\left(T, K_{L}^{*}\right) / c_{M}$ \\
\hline 2.0 & $\infty$ & 3.00 & 2 & 2.94 & 3 & 3.01 & 0 & 5.40 \\
\hline 3.0 & $\infty$ & 4.00 & 3 & 3.59 & 4 & 3.61 & 0 & 5.60 \\
\hline 4.0 & $\infty$ & 5.00 & 5 & 4.12 & 5 & 4.13 & 0 & 5.80 \\
\hline 5.0 & $\infty$ & 6.00 & 6 & 4.58 & 6 & 4.59 & 0 & 6.00 \\
\hline 6.0 & $\infty$ & 7.00 & 7 & 5.00 & 7 & 5.00 & 0 & 6.20 \\
\hline 7.0 & $\infty$ & 8.00 & 8 & 5.38 & 8 & 5.39 & 0 & 6.40 \\
\hline 8.0 & $\infty$ & 9.00 & 9 & 5.74 & 9 & 5.75 & 0 & 6.60 \\
\hline 9.0 & $\infty$ & 10.00 & 10 & 6.08 & 10 & 6.08 & 0 & 6.80 \\
\hline 10.0 & $\infty$ & 11.00 & 11 & 6.40 & 11 & 6.40 & 0 & 7.00 \\
\hline
\end{tabular}

\section{Periodic Times and Failure Number}

\subsection{Replacement First}

Suppose the unit is replaced at $N T(N=1,2, \ldots ; 0<T<\infty)$ or at failure $K(1 \leq K<\infty)$, whichever occurs first. Then, replacing formally $T$ with $N T$ in (15), the expected cost rate is

$C_{F}(N, K)=\frac{c_{T} \bar{P}_{K}(N T)+c_{K} P_{K}(N T)+c_{M} \int_{0}^{N T} \bar{P}_{K}(t) h(t) d t}{\int_{0}^{N T} \bar{P}_{K}(t) d t}$.

When $c_{T}=c_{K}$, forming the inequality $C_{F}(N+1, K)-C_{F}(N, K) \geq 0$,

$\int_{0}^{N T} \bar{P}_{K}(t)\left[H_{1}(N, K)-h(t)\right] d t \geq \frac{c_{T}}{c_{M}}$

where

$H_{1}(N, K) \equiv \frac{\int_{N T}^{(N+1) T} \bar{P}_{K}(t) h(t) d t}{\int_{N T}^{(N+1) T} \bar{P}_{K}(t) d t}$

Therefore, if $\int_{0}^{\infty} \bar{P}_{K}(t)[h(\infty)-h(t)] d t>c_{T} / c_{M}$, then there exists a finite and unique $N_{F}^{*}(1 \leq$ $N_{F}^{*}<\infty$ ) which satisfies (26), and the resulting cost rate is

$c_{M} H_{1}\left(N_{F}^{*}-1, K\right)<C_{F}\left(N_{F}^{*}, K\right) \leq c_{M} H_{1}\left(N_{F}^{*}, K\right)$.

Furthermore, (26) becomes

$N\{H[(N+1) T]-H(N T)\}-H(N T) \geq \frac{c_{T}}{c_{M}}$,

as $K \rightarrow \infty$. Thus, if $\int_{0}^{\infty} t d h(t)>c_{T} / c_{M}$, then a unique and minimum $N_{F}^{*}$ which satisfies (28) exists. Therefore, because $N_{F}^{*}$ decreases strictly with $K$ to $N^{*}$, from (27), optimum policy to minimize $C_{F}(N, K)$ is $\left(N_{F}^{*}=N^{*}, K_{F}^{*}=\infty\right)$, where $N^{*}$ is given in (28). 
International Journal of Mathematical, Engineering and Management Sciences

Vol. 3, No. 2, 136-150, 2018

https://dx.doi.org/10.33889/IJMEMS.2018.3.2-011

\subsection{Replacement Last}

Suppose the unit is replaced at time $N T(N=0,1,2, \ldots ; 0<T<\infty)$ or at failure $K(0 \leq K<$ $\infty)$, whichever occurs last. Then, replacing formally $T$ with $N T$ in (21), the expected cost rate is

$C_{L}(N, K)=\frac{c_{T} P_{K}(N T)+c_{K} \bar{P}_{K}(N T)+c_{M}\left[H(N T)+\int_{N T}^{\infty} \bar{P}_{K}(t) h(t) d t\right]}{N T+\int_{N T}^{\infty} \bar{P}_{K}(t) d t}$.

When $c_{T}=c_{K}$, forming the inequality $C_{L}(N+1, K)-C_{L}(N, K) \geq 0$,

$\int_{0}^{N T}\left[\widetilde{H}_{1}(N, K)-h(t)\right] d t-\int_{N T}^{\infty} \bar{P}_{K}(t)\left[h(t)-\widetilde{H}_{1}(N, K)\right] d t \geq \frac{c_{T}}{c_{M}}$,

where

$\widetilde{H}_{1}(N, K) \equiv \frac{\int_{N T}^{(N+1) T} P_{K}(t) h(t) d t}{\int_{N T}^{(N+1) T} P_{K}(t) d t}$.

Therefore, if $\int_{0}^{\infty} t d h(t)>c_{T} / c_{M}$, then there exists a finite and unique minimum $N_{L}^{*}\left(0 \leq N_{L}^{*}<\right.$ $\infty)$ which satisfies (30), and the resulting cost rate is

$c_{M} \widetilde{H}_{1}\left(N_{L}^{*}-1, K\right)<C_{L}\left(N_{L}^{*}, K\right) \leq c_{M} \widetilde{H}_{1}\left(N_{L}^{*}, K\right)$.

Furthermore, noting that the left-hand side of (30) increases strictly with $K$, (30) becomes (28).Therefore, because $N_{L}^{*}$ increases strictly with $K$ from $N^{*}$, from (31), optimum policy to minimize $C_{L}(N, K)$ is $\left(N_{L}^{*}=N^{*}, K_{L}^{*}=0\right)$, where $N^{*}$ is given in (28).

\subsection{Numerical Examples}

Table 7 presents optimum $N_{F}^{*}$ and $N_{L}^{*}$ and their cost rates for given $K$ when $H(t)=t^{2.0}$ and $T=0.1$. This shows that when $K=1, C_{L}\left(N_{L}^{*}, K\right)<C_{F}\left(N_{F}^{*}, K\right)$, and for $K=5$, $C_{F}\left(N_{F}^{*}, K\right)<C_{L}\left(N_{L}^{*}, K\right)$ when $c_{T} / c_{M}<5.0$.

Table 7. Optimum $N_{F}^{*}$ and $N_{L}^{*}$ and their cost rates for given $T$ when $H(t)=t^{2.0}$ and $T=0.1$

\begin{tabular}{|c|c|c|c|c|c|c|c|c|}
\hline \multirow{2}{*}{$c_{T} / c_{M}$} & \multicolumn{4}{|c|}{$K=1$} & \multicolumn{4}{c|}{$K=5$} \\
\cline { 2 - 9 } & $N_{F}^{*}$ & $C_{F}\left(N_{F}^{*}, K\right)$ & $N_{L}^{*}$ & $C_{L}\left(N_{L}^{*}, K\right)$ & $N_{F}^{*}$ & $C_{F}\left(N_{F}^{*}, K\right)$ & $N_{L}^{*}$ & $C_{L}\left(N_{L}^{*}, K\right)$ \\
\hline 2.0 & 15 & 3.38 & 14 & 2.84 & 14 & 2.83 & 16 & 3.20 \\
\hline 3.0 & 20 & 4.51 & 17 & 3.47 & 17 & 3.47 & 18 & 3.65 \\
\hline 4.0 & 21 & 5.64 & 20 & 4.00 & 20 & 4.03 & 20 & 4.09 \\
\hline 5.0 & 22 & 6.77 & 22 & 4.47 & 22 & 4.54 & 21 & 5.52 \\
\hline 6.0 & 22 & 7.90 & 24 & 4.90 & 25 & 5.02 & 24 & 4.92 \\
\hline 7.0 & 22 & 9.03 & 26 & 5.29 & 27 & 5.49 & 26 & 5.30 \\
\hline 8.0 & 23 & 10.16 & 27 & 5.66 & 27 & 5.96 & 28 & 5.66 \\
\hline 9.0 & 27 & 11.28 & 29 & 6.00 & 28 & 6.42 & 30 & 6.00 \\
\hline 10.0 & 27 & 12.41 & 30 & 6.33 & 29 & 6.88 & 30 & 6.33 \\
\hline
\end{tabular}


International Journal of Mathematical, Engineering and Management Sciences

Vol. 3, No. 2, 136-150, 2018

https://dx.doi.org/10.33889/IJMEMS.2018.3.2-011

\section{Over Time and Failure Number \\ 8.1 Replacement First}

Suppose the unit is replaced at failure $K$ or at the first failure over $T$, whichever occurs first (Nakagawa and Zhao, 2015). Then, the mean time to replacement is

$\int_{0}^{T} t d P_{K}(t)+\sum_{j=0}^{K-1} \int_{0}^{T}\left[\frac{1}{\bar{F}(t)} \int_{T}^{\infty} u d F(u)\right] d P_{j}(t)=\int_{0}^{T} \bar{P}_{K}(t) d t+\frac{\bar{P}_{K}(T)}{Q(T)}$.

The expected number of failures until replacement is

$K P_{K}(T)+\sum_{j=0}^{K-1}(j+1) p_{j}(T)=\int_{0}^{T} \bar{P}_{K}(t) h(t) d t+\bar{P}_{K}(T)=\sum_{j=0}^{K-1} P_{j}(T)$.

which agrees with (32) when $h(t)+Q(t)=1$. Then

$C_{O F}(T, K)=\frac{c_{O} \bar{P}_{K}(T)+c_{K} P_{K}(T)+c_{M} \sum_{j=0}^{K-1} P_{j}(T)}{\int_{0}^{T} \bar{P}_{K}(t) d t+\bar{P}_{K}(T) / Q(T)}$,

which agrees with $C_{O}(T)$ in (5) as $K \rightarrow \infty$ and agrees with $C_{N}(K)$ in (9) as $T \rightarrow \infty$.

When $c_{O}=c_{K}$, differentiating $C_{O F}(T, K)$ with respect to $T$ and setting it equal to zero,

$\int_{0}^{T} \bar{P}_{K}(t)[Q(T)-h(t)] d t=\frac{c_{O}}{c_{M}}$.

Thus, if $\int_{0}^{\infty} \bar{P}_{K}(t)[h(\infty)-h(t)] d t>c_{O} / c_{M}$, there exists a finite and unique $T_{O F}^{*}\left(0<T_{O F}^{*}<\right.$ $\infty)$ which satisfies (35), and the resulting cost rate is

$C_{O F}\left(T_{O F}^{*}, K\right)=c_{M} Q\left(T_{O F}^{*}\right)$

Therefore, optimum policy to minimize $C_{O F}(T, K)$ is $\left(T_{O F}^{*}=T_{O}^{*}, K_{O F}^{*}=\infty\right)$, where $T_{O}^{*}$ is given in (7). This optimum policy indicates that replacement done over time $T$ is more economical than that at failure $K$.

Next, we find optimum $K_{O F}^{*}$ to minimize $C_{O F}(T, K)$ for given $T$. Forming the inequality $C_{O F}(T, K+1)-C_{O F}(T, K) \geq 0$,

$H_{2}(T, K)\left[\int_{0}^{T} \bar{P}_{K}(t) d t+\frac{\bar{P}_{K}(T)}{Q(T)}\right]-\sum_{j=0}^{K-1} P_{j}(T) \geq \frac{c_{O}}{c_{M}}$,

where

$H_{2}(T, K) \equiv \frac{\int_{0}^{T} H(t)^{K-1} d F(t)}{\int_{0}^{T} H(t)^{K-1}\left\{\int_{t}^{\infty} e^{-[H(u)-H(t)]} d u\right\} F(t)}$.

Thus, the left-hand side of (37) increases with $K$ to that of (7). Therefore, if $T \leq T_{O}^{*}$, then $K_{O F}^{*}=\infty$, and conversely, if $T>T_{O}^{*}$, then optimum $K_{O F}^{*}\left(1 \leq K_{O F}^{*}<\infty\right)$ exists. 
International Journal of Mathematical, Engineering and Management Sciences

Vol. 3, No. 2, 136-150, 2018

https://dx.doi.org/10.33889/IJMEMS.2018.3.2-011

\subsection{Replacement Last}

Suppose that the unit is replaced at failure $K$ or at the first failure over $T$, whichever occurs last (Nakagawa and Zhao, 2015). Thus, the mean time to replacement is

$T+\int_{T}^{\infty} \bar{P}_{K}(t) d t+\frac{P_{K}(T)}{Q(T)}$

The expected number of failures is

$K \bar{P}_{K}(T)+\sum_{j=K+1}^{\infty}(j+1) p_{j}(T)=H(T)+1+\sum_{j=1}^{K-1} \bar{P}_{j}(T)$.

Eq. (39) agrees with (38) when $h(t)=Q(t)=1$. Then

$C_{O L}(T, K)=\frac{c_{O} P_{K}(T)+c_{K} \bar{P}_{K}(T)+c_{M}\left[H(T)+1+\sum_{j=1}^{K-1} \bar{P}_{j}(T)\right]}{T+\int_{T}^{\infty} \bar{P}_{K}(t) d t+\frac{P_{K}(T)}{Q(T)}}$.

which agrees with $C_{O}(T)$ in (5) as $K=0$ and agrees with $C_{N}(K)$ in (9) as $T \rightarrow 0$.

When $c_{O}=c_{K}$, differentiating $C_{O L}(T, K)$ with respect to $T$ and setting it equal to zero,

$\int_{0}^{T}[Q(T)-h(t)] d t-\int_{T}^{\infty} \bar{P}_{K}(t)[h(t)-Q(T)] d t=\frac{c_{O}}{c_{M}}$.

Thus, if $\int_{0}^{T} t d h(t)>c_{O} / c_{M}$, there exists a unique $T_{O L}^{*}\left(0<T_{O L}^{*}<\infty\right)$ which satisfies (41), and the resulting cost rate is

$C_{O L}\left(T_{O L}^{*}, K\right)=c_{M} Q\left(T_{O L}^{*}\right)$

Furthermore, letting $L_{1}(K ; T)$ be the left-hand side of $(41), L_{1}(K ; T)-L_{1}(K+1 ; T)>0$, $L_{1}(K ; T)$ decreases strictly with $K$ from the left-hand side of (7). Thus, $T_{O L}^{*}$ increases with $K$ to $T_{O}^{*}$ given in (7). Therefore, optimum policy to minimize $C_{O L}(T, K)$ is $\left(T_{O L}^{*}=T_{O}^{*}, K_{O L}^{*}=0\right)$, where $T_{O}^{*}$ is given in (7). This optimum policy indicates that replacement done over time $T$ is more economical than that at failure $K$.

Next, we find optimum $K_{O L}^{*}$ to minimize $C_{O L}(T, K)$ for given $T$. Forming the inequality $C_{O L}(T, K+1)-C_{O L}(T, K) \geq 0$,

$\widetilde{H}_{2}(T, K)\left[T+\int_{T}^{\infty} \bar{P}_{K}(t) d t+\frac{P_{K}(T)}{Q(T)}\right]-\left[H(T)+1+\sum_{j=1}^{K-1} P_{j}(T)\right] \geq \frac{c_{O}}{c_{M}}$,

where

$\widetilde{H}_{2}(T, K) \equiv \frac{\int_{T}^{\infty} H(t)^{K-1} d F(t)}{\int_{T}^{\infty} H(t)^{K-1}\left\{\int_{t}^{\infty} e^{-[H(u)-H(t)]}\right\} d F(t)}$.

Thus, the left-hand side of (43) increases with $K$ from 
International Journal of Mathematical, Engineering and Management Sciences

Vol. 3, No. 2, 136-150, 2018

https://dx.doi.org/10.33889/IJMEMS.2018.3.2-011

$\widetilde{H}_{2}(T, 1)\left[T+\frac{1}{Q(T)}\right]-H(T)-1>T Q(T)-H(T)$,

which agrees with (7). Letting $\widetilde{T}_{O}$ be a solution of

$\widetilde{H}_{2}(T, 1)\left[T+\frac{1}{Q(T)}\right]-H(T)-1=\frac{c_{O}}{c_{M}}$,

then $\widetilde{T}_{O}<T_{O}^{*}$. Therefore, if $T \geq \widetilde{T}_{O}$, then $K_{O L}^{*}=0$, and conversely, if $T<\widetilde{T}_{O}$, then optimum $K_{O L}^{*}\left(1 \leq K_{O L}^{*}<\infty\right)$ exists.

\subsection{Numerical Examples}

Table 8 presents optimum $T_{O F}^{*}$ and $T_{O L}^{*}$ and their cost rates for given $K$ when $H(t)=t^{2.0}$, This shows that when $K=5, C_{O F}\left(T_{O F}^{*}, K\right)<C_{O L}\left(T_{O L}^{*}, K\right)$ for $c_{O} / c_{M} \leq 4.0$, and $C_{O F}\left(T_{O F}^{*}, K\right)>$ $C_{O L}\left(T_{O L}^{*}, K\right)$ for $c_{O} / c_{M} \geq 5.0$.

Table 8. Optimum $T_{O F}^{*}$ and $T_{O L}^{*}$ and their cost rates for given $K$ when $H(t)=t^{2.0}$

\begin{tabular}{|c|c|c|c|c|c|c|c|c|}
\hline \multirow{2}{*}{$\begin{array}{c}c_{O} \\
/ c_{M}\end{array}$} & \multicolumn{9}{|c|}{$K=1$} & \multicolumn{4}{c|}{$K=5$} \\
\cline { 2 - 9 } & $T_{O F}^{*}$ & $\begin{array}{l}C_{O F}\left(T_{O F}^{*}, K\right) \\
/ c_{M}\end{array}$ & $T_{O L}^{*}$ & $\begin{array}{l}C_{O L}\left(T_{O L}^{*}, K\right) \\
/ C_{M}\end{array}$ & $T_{O F}^{*}$ & $\begin{array}{l}C_{O F}\left(T_{O F}^{*}, K\right) \\
/ c_{M}\end{array}$ & $T_{O L}^{*}$ & $\begin{array}{l}C_{O L}\left(T_{O L}^{*}, K\right) \\
/ C_{M}\end{array}$ \\
\hline 2.0 & 1.45 & 3.38 & 1.16 & 2.89 & 1.16 & 2.89 & 1.35 & 3.21 \\
\hline 3.0 & 2.11 & 4.51 & 1.52 & 3.50 & 1.52 & 3.50 & 1.61 & 3.66 \\
\hline 4.0 & 2.76 & 5.64 & 1.82 & 4.02 & 1.83 & 4.04 & 1.87 & 4.10 \\
\hline 5.0 & 3.44 & 6.77 & 2.09 & 4.48 & 2.12 & 4.55 & 2.11 & 4.52 \\
\hline 6.0 & 4.14 & 7.90 & 2.33 & 4.90 & 2.40 & 5.03 & 2.34 & 4.92 \\
\hline 7.0 & 4.89 & 9.03 & 2.56 & 5.29 & 2.68 & 5.49 & 2.56 & 5.30 \\
\hline 8.0 & 5.70 & 10.16 & 2.77 & 5.66 & 2.95 & 5.96 & 2.77 & 5.66 \\
\hline 9.0 & 6.59 & 11.28 & 2.97 & 6.00 & 3.22 & 6.42 & 2.97 & 6.00 \\
\hline 10.0 & 7.59 & 12.41 & 3.17 & 6.32 & 3.50 & 6.88 & 3.17 & 6.33 \\
\hline
\end{tabular}

Table 9 presents optimum $K_{O F}^{*}$ and $K_{O L}^{*}$ and their cost rates for given $T$ when $H(t)=t^{2.0}$. Noting that $1 \leq T^{*} \leq 3.16$ in Table 9 for $m=2$, when $c_{O} / c_{M}=10.0, K_{O F}^{*}=\infty$ and $K_{O L}^{*}=0$, and for $2 \leq c_{O} / c_{M} \leq 10.0$, when $T=1.0, K_{O F}^{*}=\infty$, and when $T=5, K_{O L}^{*}=0$.

Table 9. Optimum $K_{O F}^{*}$ and $K_{O L}^{*}$ and their cost rates for given $T$ when $H(t)=t^{2.0}$

\begin{tabular}{|c|c|c|c|c|c|c|c|c|}
\hline \multirow{2}{*}{$\begin{array}{l}c_{O} \\
/ c_{M}\end{array}$} & \multicolumn{4}{|c|}{$T=1.0$} & \multicolumn{4}{c|}{$T=5.0$} \\
\hline 2.0 & $K_{O F}^{*}$ & $\begin{array}{l}C_{O F}\left(T, K_{O F}^{*}\right) \\
/ c_{M}\end{array}$ & $K_{O L}^{*}$ & $\begin{array}{l}C_{O L}\left(T, K_{O L}^{*}\right) \\
/ c_{M}\end{array}$ & $K_{O F}^{*}$ & $\begin{array}{l}C_{O F}\left(T, K_{O F}^{*}\right) \\
/ c_{M}\end{array}$ & $K_{O L}^{*}$ & $\begin{array}{l}C_{O L}\left(T, K_{O L}^{*}\right) \\
/ c_{M}\end{array}$ \\
\hline 3.0 & $\infty$ & 2.89 & 0 & 2.89 & 2 & 3.01 & 0 & 5.48 \\
\hline 4.0 & $\infty$ & 4.62 & 3 & 3.58 & 3 & 3.61 & 0 & 5.68 \\
\hline 5.0 & $\infty$ & 5.07 & 4 & 4.12 & 4 & 4.13 & 0 & 5.87 \\
\hline 6.0 & $\infty$ & 5.79 & 6 & 4.58 & 5 & 4.59 & 0 & 6.07 \\
\hline 7.0 & $\infty$ & 6.52 & 7 & 5.00 & 6 & 5.00 & 0 & 6.26 \\
\hline 8.0 & $\infty$ & 7.24 & 8 & 5.75 & 7 & 5.39 & 0 & 6.46 \\
\hline 9.0 & $\infty$ & 7.96 & 9 & 6.08 & 9 & 6.08 & 0 & 6.66 \\
\hline 10.0 & $\infty$ & 8.69 & 10 & 6.40 & 10 & 6.40 & 0 & 7.05 \\
\hline
\end{tabular}


International Journal of Mathematical, Engineering and Management Sciences

Vol. 3, No. 2, 136-150, 2018

https://dx.doi.org/10.33889/IJMEMS.2018.3.2-011

\section{Conclusions}

We have summarized several extended replacement models with number of failures in this paper: (i) The standard periodic replacement model with times $n T$ in (Barlow and Proschan, 1965; Nakagawa, 2005); (ii) Replacement is done at the first failure over $T$. (iii) Replacement is done at failure number $K$. (iv) Replacement is done at the next periodic time overt periodic times $n T$ when more than $K$ failures have arrived. (v) Replacement is done at time $T$ or at failure number $K$, whichever occurs first, or whichever occurs last. (vi) Replacement is done at time $N T$ or at failure number $K$, whichever occurs first, or whichever occurs last. (vii) Replacement is done at failure number $K$ or at the first failure over $T$, whichever occurs first, and whichever occurs last. Decision variables $T, N$ and $K$ of replacement policies have been optimized analytically and computed numerically.

It has been shown that optimum time in (ii) is less than or equal to that in (i) and the policy in (i) is more economical than that in (ii). When the number of failures $K$ has been considered in (iii), its comparison with (i) is discussed from the optimization in (v), which indicates that replacement done at $T$ is more economical than that at $K$. The overtime replacement in (vii) also shows that replacement done at failure $K$ is not so economical. Comparisons of the approaches of whichever occurs first and last in (v), (vi) and (vii) indicates that both have advantage and disadvantage in cost, and the analytical discussions show that we can compare the replacement policies from the optimizations of their integrated models.

\section{Acknowledgement}

This paper was made possible by National Natural Science Foundation of China under Grant No. 71371097.

\section{References}

Barlow, R. E., \& Proschan, F. (1996). Mathematical theory of reliability. Society for Industrial and Applied Mathematics.

Fuqing, Y., \& Kumar, U. (2012). A general imperfect repair model considering time-dependent repair effectiveness. IEEE Transactions on Reliability, 61(1), 95-100.

Golmakani, H. R., \& Moakedi, H. (2012). Periodic inspection optimization model for a two-component repairable system with failure interaction. Computers \& Industrial Engineering, 63(3), 540-545.

Huynh, K. T., Barros, A., Berenguer, C., \& Castro, I. T. (2011). A periodic inspection and replacement policy for systems subject to competing failure modes due to degradation and traumatic events. Reliability Engineering \& System Safety, 96(4), 497-508.

Huynh, K. T., Castro, I. T., Barros, A., \& Berenguer, C. (2012). Modeling age-based maintenance strategies with minimal repairs for systems subject to competing failure modes due to degradation and shocks. European Journal of Operational Research, 218(1), 140-151.

Kim, D. K., Lim, J. H., \& Park, D. H. (2015). Optimal maintenance level for second-hand product with periodic inspection schedule. Applied Stochastic Models in Business and Industry, 31(3), 349-359.

Nakagawa, T. (2005). Maintenance theory of reliability. Springer Science \& Business Media.

Nakagawa, T., \& Zhao, X. (2015). Maintenance overtime policies in reliability theory, Springer, Switzerland.

Park, M., Jung, K. M., \& Park, D. H. (2013). Optimal post-warranty maintenance policy with repair time threshold for minimal repair. Reliability Engineering \& System Safety, 111, 147-153. 
International Journal of Mathematical, Engineering and Management Sciences

Vol. 3, No. 2, 136-150, 2018

https://dx.doi.org/10.33889/IJMEMS.2018.3.2-011

Pulcini, G. (2003). Mechanical reliability and maintenance models. In Handbook of Reliability Engineering, Springer London, 317-348.

Taghipour, S., \& Banjevic, D. (2012). Optimum inspection interval for a system under periodic and opportunistic inspections. IIE Transactions, 44(11), 932-948.

Taghipour, S., \& Kassaei, M. L. (2015). Periodic inspection optimization of a k-out-of-n load-sharing system. IEEE Transactions on Reliability, 64(3), 1116-1127.

Taghipour, S., Banjevic, D., \& Jardine, A. K. (2010). Periodic inspection optimization model for a complex repairable system. Reliability Engineering \& System Safety, 95(9), 944-952.

Toledo, M. L. G. D., Freitas, M. A., Colosimo, E. A., \& Gilardoni, G. L. (2016). Optimal periodic maintenance policy under imperfect repair: A case study on the engines of off-road vehicles. IIE Transactions, 48(8), 747-758.

Venkatesh, J. (2007). An introduction to total productive maintenance (TPM). The Plant Maintenance Resource Center, 3-20.

Wang, W., \& Banjevic, D. (2012). Ergodicity of forward times of the renewal process in a block-based inspection model using the delay time concept. Reliability Engineering \& System Safety, 100, 1-7.

Zhao, X., \& Nakagawa, T. (2012). Optimization problems of replacement first or last in reliability theory. European Journal of Operational Research, 223(1), 141-149.

Zhao, X., Al-Khalifa, K. N., Hamouda, A. M., \& Nakagawa, T. (2016). First and last triggering event approaches for replacement with minimal repairs. IEEE Transactions on Reliability, 65(1), 197-207.

Zhao, X., Mizutani, S., \& Nakagawa, T. (2015). Which is better for replacement policies with continuous or discrete scheduled times? European Journal of Operational Research, 242(2), 477-486. 